\title{
ADIPONECTIN LEVEL AND ITS RELATION TO OSTEOPOROSIS IN PATIENTS WITH RHEUMATOID ARTHRITIS
}

\author{
Randa Mohammed youssef ${ }^{* *}$, Mona El-Sebaie*, Neven Mahmoud Taha* and \\ Howayda Farouk Zidan*
}

\author{
*Physical Medicine, \\ Rheumatology and \\ RehabilitationFaculty of \\ Medicine, Ain-Shams \\ University,Egypt \\ Corresponding author \\ Randa Mohammed youssef \\ Mobile: \\ E.mail: \\ randayoussef510@gmail.com
}

Received: $25 / 3 / 2021$

Accepted: 29/4/2021

\section{Online ISSN: 2735-3540}

\begin{abstract}
Background: Adiponectin possesses anti-inflammatory properties and also has pro-inflammatory aspects in some diseases. Adiponectin plays an important role in bone metabolism and osteoporosis (OP) pathophysiology, modulating bone formation and remodeling by selectively enhancing stem cell development between adipocyte and bone precursors in the bone marrow in response to reduced energy storage and metabolic needs.
\end{abstract}

Aim of work: The aim of our study was to assess serum adiponectin level in rheumatoid arthritis (RA) patients and to study the possible role of adiponectin as a marker of osteoporosis in rheumatoid arthritis patients.

Patient and Methods: The study included 30 premenopausal rheumatoid arthritis female patients. The age ranged from 26 to 46 years old and body mass index (BMI) ranged from 23 to 37.34 compared to 15 healthy controls. They were subjected to full history taking. Laboratory investigation was done to exclude other causes of osteoporosis. Serum level of adiponectin was measured by ELISA. They were also assessed radiologically by DEXA scan of lumbar spine to confirm osteoporosis.

Results: In our study, we found that RA patients had statistically significant higher adiponectin level than healthy controls. Moreover, serum adiponectin was significantly higher in patients with both RA and OP compared to RA female patients only. Moreover, our results demonstrated that a serum adiponectin of $1400 \mu \mathrm{g} / \mathrm{L}$ or higher is capable of detecting osteoporosis among RA patients with a sensitivity of $100 \%$ and a specificity of 100\%. Regarding predictive ability of adiponectin in detecting RA with osteoporosis, our findings showed that there was an inverse association between adiponectin level and bone density, and there was a significant negative correlation between adiponectin level and DEXA scores in RA group.

Conclusion: There was a clinical usefulness of measuring serum adiponectin in predicting $O P$ among $R A$ premenopausal female patients. This was strongly suggest the possible utility of serum adiponectin as a marker to detect $O P$ in patients with RA hand in hand with conventional parameters.

Keywords: Adiponectin, Osteoporosis, Rheumatoid Arthritis.

\section{INTRODUCTION:}

Rheumatoid arthritis (RA) is a chronic autoimmune disease characterized by synovial inflammation often followed by cartilage and bone erosion. The patients suffer from reduced functional ability, pain, 
and stiffness that often lead to impaired quality of life. The prevalence of RA is $\sim 0.5-1 \%$ of the western population and the disease is more common among women and elderly ${ }^{(1)}$.

Osteoporosis (OP) is one of the most common comorbidities associated with RA, a risk factor for osteoporosis, is accompanied by both periarticular and systemic osteoporosis. The mechanisms of osteoporosis associated with RA involve the influence of inflammatory cytokines, such as interleukin IL-1, IL-6, and tumor necrosis factor (TNF); glucocorticoid treatment; and immobility ${ }^{(2)}$.

Adiponectin possesses antiinflammatory properties and also has proinflammatory aspects in some diseases. Adiponectin functions as an insulin sensitizer and exhibits anti-diabetic, antiinflammatory, and anti-atherogenic effects. Its multifunctional aspects render it a highly favorable target for metabolic disorders. The central role of adiponectin is energy homeostasis with a newly proposed role as a "starvation gene" $(3,4)$.

Adiponectin plays an important role in bone metabolism and osteoporosis pathophysiology, modulating bone formation and remodeling by selectively enhancing stem cell development between adipocyte and bone precursors in the bone marrow in response to reduced energy storage and metabolic needs ${ }^{(5)}$.

\section{AIM OF THE WORK:}

The aim of this study is to assess serum adiponectin level in RA patients and to study the possible role of adiponectin as a marker of osteoporosis in RA patients.

\section{PATIENTS AND METHODS:}

The present study included 30 premenopausal RA female patients fulfilled the ACR/ EULAR 2010 classification criteria for RA patients and 15 healthy females age and BMI matched as a control group.

\section{Inclusion criteria:}

Pre-menopausal female RA patients (ranged from 26 to 46 years) diagnosed according to the 2010 American College of Rheumatology (ACR)/European League against Rheumatism (EULAR) RA classification criteria (ACR/ EULAR, 2010).

\section{Exclusion criteria:}

Pregnancy, breast feeding female, patients with other causes of secondary osteoporosis as smoking, bed ridden, hyperthyroidism ,primary hyperparathyrodisim , malignancy, malabsorption, osteomalacia and cushing's syndrome, patients on treatment of osteoporosis (Anti resorptive drugs and bone forming "anabolic" drugs), morbid obesity (BMI more than or equal 40) and patients on drugs that may influence bone metabolism (estrogen, glucocorticoids, anticonvulsants, vitamin $\mathrm{D}$, calcium); all of these cases were excluded from our study.

- Written consent was taken from all patients, the study was approved by Medical Ethics Committee.

\section{All patients will undergo the following:}

1. Full medical history taking with special attention to history of previous fracture, calcium, vitamin D or anti-resorptive medications

2. Full Clinical examination

3. Laboratory investigation including complete blood count, Erythrocyte sedimentation rate, rheumatoid factor, Anti cyclic citrullinated peptide, Liver function tests, Kidney function tests and Serum adiponectin level $(\mu \mathrm{g} / \mathrm{ml})$ using Human Adiponectin ELISA commercial test system.

4. Radiological assessment: DUAL Energy X-ray Absorptiometry (DEXA). 
Both patients and controls are subjected to:

A- Measurement of Serum adiponectin level $(\mu \mathrm{g} / \mathrm{ml})$ using Human Adiponectin ELISA commercial test system.

B- Radiological assessment of Bone Mineral Density in lumber spine (L2L4) by using DEXA which categorizes the osteoporosis according to T-score: Normal (-1.0 and above), osteopenia (1.0 to -2.5$)$, osteoporosis $(-2.5$ and below), "established" osteoporosis ( -2.5 and below plus one or more osteoporotic fracture(s).

\section{Statistical Analysis:}

1. Student $T$ test was used to assess the statistical significance of the difference between two study group means.

2. Chi-Square test was used to examine the relationship between two qualitative variables

3. The ROC Curve (Receiver Operating Characteristic) provides a useful way to evaluate the Sensitivity and specificity for quantitative Diagnostic measures that categorize cases into one of two groups

4. Correlation analysis (using Pearson's method): To assess the strength of association between two quantitative variables. The correlation coefficient denoted symbolically " $r$ " defines the strength (magnitude) and direction (positive or negative) of the linear relationship between two variables.

- $\mathrm{r}=0-0.19$ is regarded as very weak correlation

- $\mathrm{r}=0.2-0.39$ as weak correlation

- $\mathrm{r}=0.40-0.59$ as moderate correlation

- $\mathrm{r}=0.6-0.79$ as strong correlation.

- $\mathrm{r}=0.8-1$ as very strong correlation.

\section{RESULTS:}

The present study included 30 premenopausal RA female patients and 15 healthy female. There was no statistically significant difference between RA patients and controls regarding age and BMI as shown in table (1).

There was statistically highly significant increase in the level of adiponectin in RA patients compared to controls (P-value $<0.001)$ as shown in table (2) \& diagram (1).

As regard DEXA T-score at lumbar spine (L1-L4) there was statistically highly significant difference (P-value <0.001) between RA patients and controls as shown in table (3).

RA patients were classified as regard the presence of OP to group A (RA patients without OP) and group B (RA patients with OP)

There was no statistical significant difference between group A and group B as regards age $(\mathrm{P}$-value $=0.455)$.

There was statistically significant increase in BMI in group (A) patients compared to group B (P-value <0.001) and there was no statistical significant difference between both groups as regards disease duration (P-value $=0.898$ ) as in table (4).

There was statistically highly significant increase in serum adiponectin in group $\mathrm{B}(\mathrm{P}$ value $<0.001)$ as in table (5) \& diagram (2).

As regards T-score at lumbar spine; there was statistically highly significant increase in $\mathrm{T}$ score in group B compared to group A (P-value $<0.001)$ as in table (6) \& diagram (3).

There was a significant negative correlation between adiponectin level and DEXA in group A and B patients (RA patients $) \quad(r=\mathbf{- 0 . 6 3 5} \& \quad r=\mathbf{- 0 . 6 0 6}$ respectively) with a statistically significant 
$\mathrm{p}$-value ( $\mathrm{p}=0.011 \& \mathrm{p}=0.017$ respectively) as in table (7) \& diagram (4).

The ROC curve was used to define the best cut-off value of serum adiponectin between group A and group B.

The cut-off value of serum adiponectin level $>\mathbf{6 0 0}$ to detect rheumatoid patient with area under curve (AUC) 1 (95\% confidence Interval $(\mathrm{CI})=0.921$ to $1.000, \mathrm{p}$ value $<0.001), \quad$ sensitivity $100 \%$ and specificity $100 \%$ as shown in table (8).

The cut-off value of serum adiponectin level $>\mathbf{1 4 0 0}$ to detect osteoporosis in patients with RA with area under curve (AUC) 1 (95\% confidence Interval $(\mathrm{CI})=0.841$ to 1.000 , p value $<0.001$ ), sensitivity of $100 \%$ and specificity of $100 \%$, as shown in table (9).

Table (1): Comparison between RA patients and controls as regard age and BMI.

\begin{tabular}{|c|c|c|c|c|c|c|}
\hline \multirow{2}{*}{} & \multicolumn{2}{|c|}{ RA patients $(\mathrm{n}=30)$} & \multicolumn{2}{c|}{ Controls $(\mathrm{n}=15)$} & \multicolumn{2}{c|}{$\mathrm{t}$ test } \\
\cline { 2 - 7 } & Range & Mean \pm SD & Range & Mean \pm SD & p value & sig. \\
\cline { 2 - 7 } & $26-46$ & $37.2 \pm 6.9$ & $26-46$ & $35.9 \pm 6.8$ & 0.554 & NS \\
\hline Age $(\mathrm{y})$ & $23-37.34$ & $29.85 \pm 4.06$ & $23-37.79$ & $28.79 \pm 3.11$ & 0.338 & NS \\
\hline
\end{tabular}

BMI: Body mass index

Table (2): Comparison between RA patients and controls groups in serum adiponectin level.

\begin{tabular}{|c|c|c|c|c|c|c|}
\hline & \multicolumn{2}{|c|}{ RA patients(n:30) } & \multicolumn{2}{|c|}{ Control(n:15) } & \multicolumn{2}{|c|}{$\mathrm{t}$ test } \\
\hline \multirow{2}{*}{$\begin{array}{c}\text { Adiponectin } \\
\text { level } \\
(\text { microgm/L) }\end{array}$} & Range & Mean \pm SD & Range & Mean \pm SD & $\mathrm{p}$ value & sig. \\
\hline & $750-3000$ & $1574.0 \pm 565.4$ & $175-300$ & $345.0 \pm 153.6$ & $<0.001$ & $\mathrm{HS}$ \\
\hline
\end{tabular}

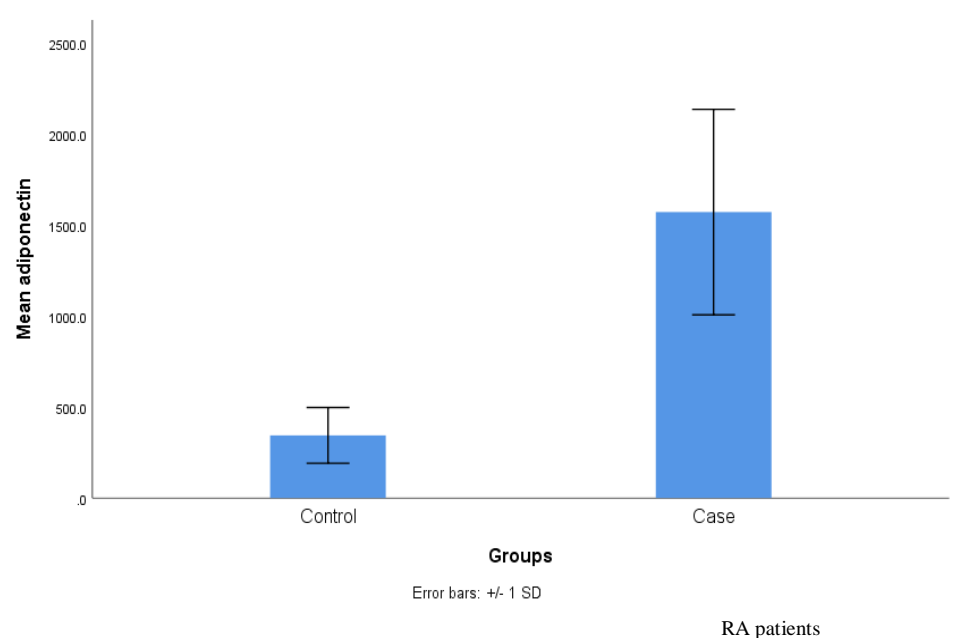

Diagram (1) Comparison between RA patients and controls groups in serum aaiponectin level

Table (3): Comparison between RA patients and controls as regards T-score at the lumbar spine.

\begin{tabular}{|c|c|c|c|c|c|c|}
\hline \multirow{2}{*}{} & \multicolumn{2}{|c|}{ RA patients (n:30) } & \multicolumn{2}{c|}{ Control (n:15) } & \multicolumn{2}{c|}{$\mathrm{t}$ test } \\
\cline { 2 - 7 } & Range & Mean \pm SD & Range & Mean \pm SD & $\mathrm{p}$ value & sig. \\
\hline DEXA & $(-3.9)-2.1$ & $-1.1 \pm 2.2$ & $0.8-2.5$ & $1.5 \pm 0.5$ & $<0.001$ & HS \\
\hline
\end{tabular}


Table (4): Comparison between group A and group B as regards descriptive data

\begin{tabular}{|c|c|c|c|c|c|c|}
\hline \multirow{2}{*}{} & \multicolumn{2}{|c|}{ RA patients without OP } & \multicolumn{2}{c|}{ RA patients with OP } & \multicolumn{2}{c|}{$\mathrm{t}$ test } \\
\cline { 2 - 7 } & Range & Mean \pm SD & Range & Mean \pm SD & p value & sig. \\
\hline Age $(\mathrm{y})$ & $27-46$ & $38.2 \pm 6.5$ & $26-45$ & $36.3 \pm 7.5$ & 0.455 & NS \\
\hline BMI $(\mathrm{kg} / \mathrm{m} 2)$ & $24.8-37.97$ & $31.54 \pm 3.96$ & $23-37$ & $28.16 \pm 3.51$ & 0.020 & $\mathrm{~S}$ \\
\hline $\mathrm{DD}(\mathrm{Y})$ & $1-13$ & $7.47 \pm 3.02$ & $4-12$ & $7.60 \pm 2.61$ & 0.898 & $\mathrm{NS}$ \\
\hline
\end{tabular}

BMI: Body mass index. DD: Disease duration.

Table (5): Comparison between group A and B patients as regards serum adiponectin level.

\begin{tabular}{|c|c|c|c|c|c|c|}
\hline & \multicolumn{2}{|c|}{ RA patients without OP } & \multicolumn{2}{c|}{ RApatients with OP } & \multicolumn{2}{c|}{$\mathrm{t}$ test } \\
\cline { 2 - 7 } & Range & Mean \pm SD & Range & Mean \pm SD & p value & sig. \\
\hline $\begin{array}{c}\text { adiponect } \\
\text { in }\end{array}$ & $750-1400$ & $1155.0 \pm 215.3$ & $1500-3000$ & $1993.0 \pm 489.6$ & $<0.001$ & HS \\
\hline
\end{tabular}

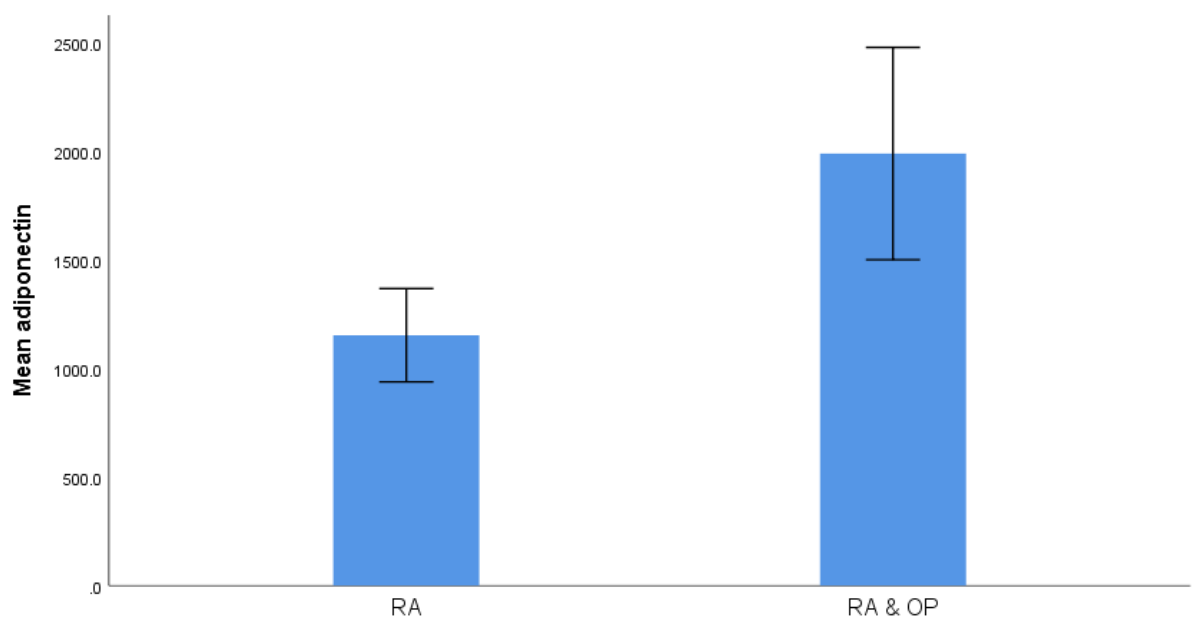

Error bars: $+1-1 \mathrm{SD}$

Diagram (2): Comparison between group A and B patients as regards serum adiponectin level.

Table (6):Comparison between group A and group B as regards T-score at the lumbar spine.

\begin{tabular}{|c|c|c|c|c|c|c|}
\hline \multirow{2}{*}{} & \multicolumn{2}{|c|}{ RA patients without OP } & \multicolumn{2}{c|}{ RApatients with OP } & \multicolumn{2}{c|}{ t test } \\
\cline { 2 - 7 } & Range & Mean \pm SD & Range & Mean \pm SD & p value & sig. \\
\hline DEXA & $(0.1)-(2.1)$ & $1.0 \pm 0.7$ & $(-3.9)-(-2.5)$ & $-3.1 \pm 0.5$ & $<0.001$ & HS \\
\hline
\end{tabular}




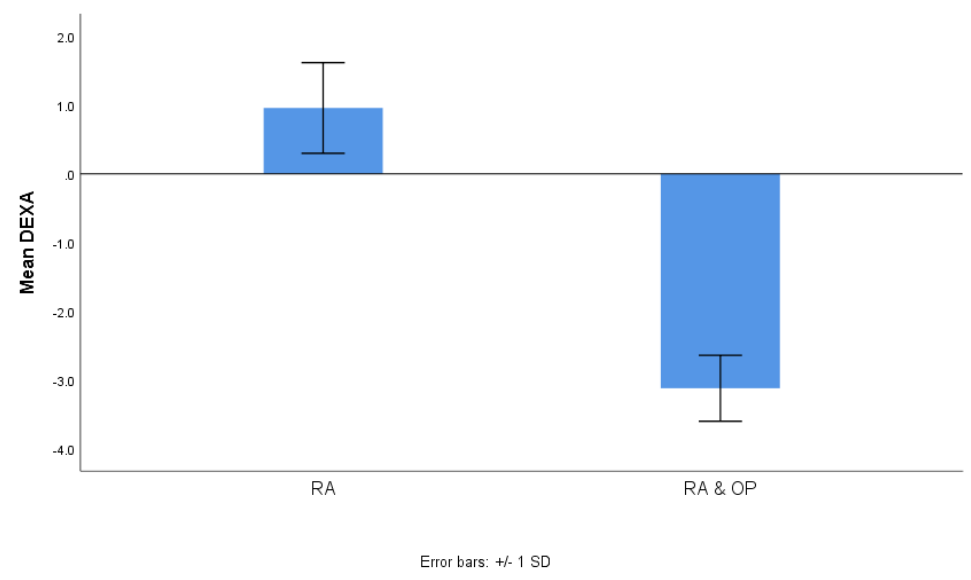

Diagram (3): Comparison between group A and group B as regards T-score at the lumbar spine.

Table (7): Correlation between serum adiponectine and T-score in groups (A\&B)

\begin{tabular}{|c|c|c|c|c|}
\hline \multirow{3}{*}{ Groups } & \multicolumn{2}{|c|}{ RA patients without OP } & \multicolumn{2}{c|}{ RA patients with OP } \\
\cline { 2 - 5 } & \multicolumn{2}{|c|}{ T-score } & \multicolumn{2}{c|}{ T-score } \\
\cline { 2 - 5 } & $\mathrm{r}$ & $\mathrm{p}$ value & $\mathrm{r}$ & $\mathrm{p}$ value \\
\hline adiponectin & $\mathbf{- 0 . 6 3 5}$ & $\mathbf{0 . 6 0 6}$ & $\mathbf{0 . 0 1 7}$ \\
& & $\mathbf{0 . 0 1 1}$ & & $\mathbf{0 . 0}$ \\
\hline
\end{tabular}

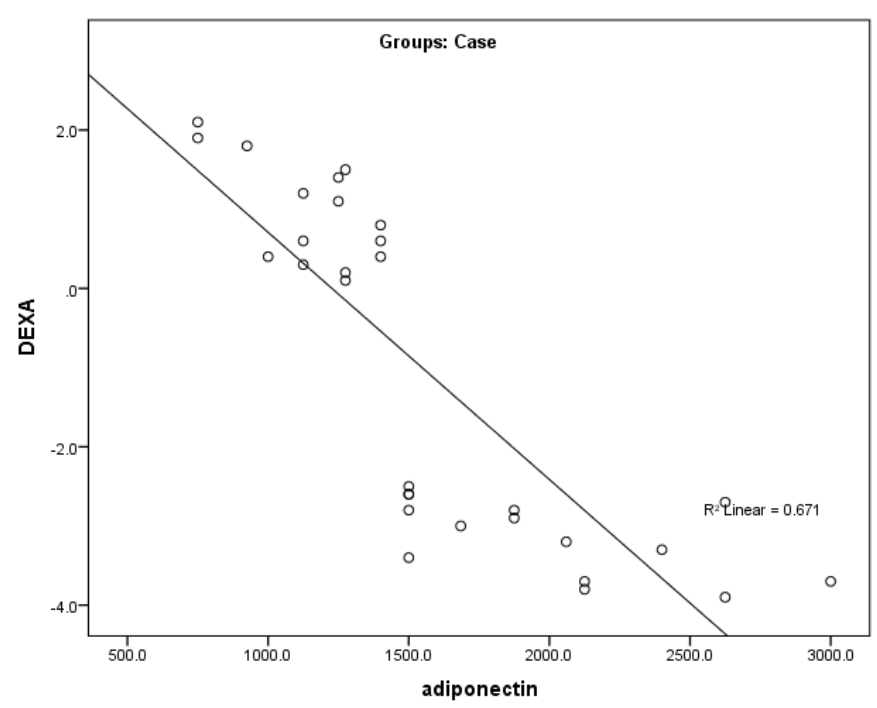

Diagram(4): Correlation between serum adiponectine and T-score in groups (A\&B).

Table (8): The area under the ROC curve (AUC) for serum adiponectin in Rheumatoid patients.

\begin{tabular}{|c|c|c|c|c|c|c|c|c|}
\hline AUC & $95 \%$ CI & p value & sig. & Cut off point & Sensitivity & Specificity & +PV & -PV \\
\hline 1 & 0.921 to 1.000 & $<0.001$ & S & $>600$ & 100 & 100 & 100 & 100 \\
\hline
\end{tabular}

AUC: Area under curve, CI: confidence Interval, PV: predictive value 
Table (9): The area under the ROC curve (AUC) for serum adiponectin level to detect patients with RA \& OP.

\begin{tabular}{|c|c|c|c|c|c|c|c|c|}
\hline AUC & $95 \%$ CI & p value & sig. & Criterion & Sensitiviy & Specifity & + PV & -PV \\
\hline 1 & 0.841 to 1.000 & $<0.001$ & $\mathrm{~S}$ & $>1400$ & 100 & 100 & 100 & 100 \\
\hline
\end{tabular}

AUC: Area under curve, CI: confidence Interval, PV: predictive value.

\section{DISCUSSION:}

Osteoporosis is a condition characterized by low bone mass and decreased bone strength, resulting in an increased propensity for fracture. OP is one of the major comorbidities of RA and is caused by a number of complex pathophysiologic processes ${ }^{(6)}$. Adiponectin is predominantly produced by adipose tissue and is adipocyte-specific adipocytokine. It is a specific protein secreted by adipocytes with presumptive anti-atherogenic and antiinflammatory characteristics. It is considered to have multiple beneficial effects on metabolism including insulin-sensitizing, anti-inflammatory, and antiapoptotic effects $^{(7)}$.

In our study we had 30 RA patients, they were divided into 2 groups according to DEXA results group (A) RA without OP and group (B) patients RA\&OP. In the present study, the mean of serum adiponectin level in the RA patients was (1574 \pm 565.4$)$ while in the controls was $(345 \pm 153.6)$ with statistically highly significant increase in RA patients compared to controls ( $p$ value $<0.001)$.

In consistent with our findings, a study conducted by Polyakova et al., $2017^{(8)}$ on 88 RA women and 45 healthy females,serum adiponectin level was statistically significant higher in RA females compared to healthy controls $(p<0.001)$. Likewise, Qian and his colleagues $^{(9)}$ in 2018 studied the serum levels of adiponectin in 38 patients with RA, 40 patients with osteoarthritis (OA), and 20 healthy controls and they found that adiponectin level was significantly higher in RA group compared to healthy controls.

The same result was recorded by Nicolaou et al.,2018 ${ }^{(10)}$ who found that serum adiponectin was significantly higher in RA patients at baseline; however, its level tends to reach normal levels as DAS -28 score improved over time.

Also, A recent meta-analysis investigated 813 RA patients and 684 healthy controls in 11 studies and indicated that circulating adiponectin level of RA patients was significantly higher than that in control groups ${ }^{(11)}$.

All these studies recorded the higher adioponectin level in RA patients as adiponectin possesses pleiotropic effects on chronic inflammatory diseases. Also, increase adiponectin in the synovium promotes higher yield secretion of chemical mediators (IL1, IL6, IL8 ,MCPand MMPs) from RA synovial fibroblasts and induces lymphocytes to synthesize TNFa,IL6 and IL8 ${ }^{(12)}$.

In contrast with our study $\mathrm{Li}$ et al., $2015^{(13)}$ who studied the serum adiponectin level in established $180 \mathrm{RA}$ Chinese female patients and 160 age- and sexmatched healthy volunteers ,they reported lower adiponectin levels in RA patients compared to healthy controls. Also Veluri and his colleagues ${ }^{(14)}$ in 2019 studied forty RA patients (newly diagnosed $n=20$, RA in clinical remission $n=20$ ) and thirty healthy controls and found that serum adiponectin 


\section{Randa Mohammed youssef, et al.,}

levels were significantly decreased in groups of RA patients .

A recent study conducted by Zhao et al., ${ }^{(15)}$ in 2020 measured serum adiponectin in 617 patients with RA and 639 healthy controls they found that patients with RA had considerably lower plasma level of adiponectin.

The difference in these results suggests that the actual relationship of adiponectin with inflammatory milieu of RA is quite complex. Physiologically, adiponectin inhibits the release of pro-inflammatory cytokines and increase the production of anti-inflammatory cytokines from activated inflammatory cells. Conversely, pro-inflammatory mediators such as TNF- $\alpha$ and IL-6 are known to suppress adiponectin production ${ }^{(16)}$.

Also, this difference can be explained by the presence of different adiponectin isoforms, there are 3 isoforms (HMW, MMW, gAPN). Many studies recorded higher HMW and gAPN in patients with RA while MMW showed no difference in RA patients compared to controls, so different isoforms of adiponectin may be the cause of different results recorded in these studies ${ }^{(4)}$.

Other reasons why adiponectin concentrations had shown variable results between studies include variable disease duration, disease activity, sample size, and ethnicity of the study population ${ }^{(11)}$.

Lumbar spine bone densitometry assessed by DEXA is the standard technique to determine secondary osteoporosis in RA, In our study the T score at lumbar spine (L1L4) for RA patients ranged between -3.9 to 2.1 with mean $(-1.1 \pm 2.2)$, and for the controls T-score at lumbar spine (L1-L4) was ranging between 0.8 to 2.5 with mean $(1.5 \pm 0.5)$ which was highly significant and $50 \%$ of our patients had OP.

However our results differ from a study conducted by Fassio et al ., 2016 ${ }^{(17)}$ who reported that, among women with RA, the proportion of pre-menopausal patients with OP was $13 \%$ compared with healthy women, This difference may be due to difference in sample size, disease duration and disease activity and ethnicity of the study population.

In our study the serum level of adiponectin was significantly higher in patients with both RA and OP compared to RA patients only $(\mathrm{p}<0.001)$. In agreement with our result, Polyakova et al., 2017 ${ }^{(8)}$ reported that RA patients with overlying OP disease had significantly higher serum adiponectin than patients who suffers RA with normal bone density $(p<0.001)$. The same result was recorded by Mpalaris and his colleagues ${ }^{(21)}$ in 2016 who studied the relationship between adiponectin and OP and found that women with OP had higher adiponectin concentrations compared to those without OP.

One of our strengths was identifying a new predictive cut-off value of serum adiponectin level $>600 \mu \mathrm{g} / \mathrm{L}$ to detect RA patient, with a sensitivity of $100 \%$ and a specificity of $100 \%$. To our knowledge no other studies reported cut-off value of serum adiponectin to diagnose RA.

Our results confirm that a serum adiponectin of $1400 \mu \mathrm{g} / \mathrm{L}$ or higher is capable to detect osteoporosis among RA patients with a sensitivity of $100 \%$ and a specificity of $100 \%$.Also Polyakova et al., ${ }^{(8)}$ reported the first cut-off value for predicting OP among RA patients which was $\geq 44 \mu \mathrm{g} /$ $\mathrm{ml}$.

However, our study faced some limitations, first the patients number were small,second we couldn't judge the effect of different RA treatment on serum adiponectin level. Third,we didn't monitor serum adiponectin level serially to detect the changes in its level with the course of the disease.Nevertheless, these limitations didn't detract from a potentially valuable role of serum adiponectin in detection $\mathrm{OP}$ in 
patients with RA as suggested by the results of our study.

In conclusion we demonstrated that there was clinical usefulness of measuring serum adiponectin in predicting osteoporosis among premenopause RA female patients which strongly suggest the possible utility of serum adiponectin as a marker to detect OP in patients with RA hand in hand with conventional parameters.

We recommend RA patients should be routinely screened for the presence of osteoporosis, Serum adiponectin can be used as a marker of osteoporosis in rheumatoid patients together with DEXA. Further longitudinal studies with larger numbers of patients are recommended to detect changes in serum adiponectin level during the course of the disease. Further studies are recommended to detect the effect of treatment of RA patients on serum adiponectin level and further studies are recommended to detect the relation between DEXA on head of the femur in RA patients and serum adiponectin level.

\section{Declaration of interests:}

The authors declared no conflicits of interest with respect to the authorship and/or publication of this article.

\section{Support:}

The research was not funded. Costs were the responsibility of the authors and instruments used in the study belong to Faculty of medicine, a part of Ain shams University, which is a public governmental organization. The authors indicate no relationship/ conditions/ circumstances that present a potential financial conflict of interest. No other financial disclosures

\section{REFERANCES:}

1. Vadell A, Bärebring L, Hulander E, Gjertsson I, Lindqvist HM and Winkvist A (2020):Anti-inflammatory Diet in Rheumatoid Arthritis (ADIRA)-a randomized, controlled crossover trial indicating effects on disease activity. The American journal of clinical nutrition; 111(6):1203-1213.

2. Wilson JC, Sarsour K, Gale S, PethöSchramm A, Jick SS and Meier CR (2019):Incidence and risk of glucocorticoidassociated adverse effects in patients with rheumatoid arthritis. Arthritis Care Res; (71):498-511.

3. Frankenberg ADV, Reis AF and Gerchman F (2017): Relationships between adiponectin levels, the metabolic syndrome, and type 2 diabetes: : a literature review. Arch Endocrinol Metab; 61(6):614-622.

4. Choi HM, Doss HM and Kim KS (2020): Multifaceted Physiological Roles of Adiponectin in Inflammation and Diseases.International journal of molecular sciences; 21(4): 1219.

5. Suh YJ , McDonald MN, Washko GR , Carolan BJ, Bowler RP, Lynch DA, Kinney GL, Bon JM, Cho MH, Crapo JD and Regan EA (2018):COPDGene Investigators Lung, Fat and Bone: Increased Adiponectin Associates with the Combination of Smoking-Related Lung Disease and Osteoporosis.Chronic obstructive pulmonary diseases (Miami, Fla.); 5(2):134-143.

6. Adami G and Saag KG (2019): Osteoporosis Pathophysiology, Epidemiology, and Screening in Rheumatoid Arthritis. Current Rheumatology Reports; 7(21): 34.

7. Geagea AG, Mallat S, Matar CF, Zerbe R, Filfili E, Francis M, Haidar H and Jurjus A (2018) :Adiponectin and Inflammation in Health and Disease: An Update. Open Medicine Journal, 5(1).

8. Polyakova Y, Sivordova L, Akhverdyan Y, Kravtcov V and Zavodovsky (2017):New biomarker for diagnosis of osteoporosis in rheumatoid arthritis patients. Postgraduate Medical Journal; (96):149-155.

9. Qian J, Xu L, Sun X, Wang Y, Xuan W, Zhang Q, Zhao P, Wu Q, Liu R, Che N, Wang $F$, Tan $W$ and Zhang $M$ (2018):Adiponectin aggravates bone 
erosion by promoting osteopontin production in synovial tissue of rheumatoid arthritis. Arthritis Research \& Therapy; 1(20): 26.

10. Nicolaou N, Joseph $\mathrm{J}$ and Bruce I. N (2018). AB0277 Leptin and adiponectin levels in rheumatoid arthritis patients.

11. Lee YA, Hahm DH, Kim JY, Sur B, Lee HM, Ryu CJ, Yang HI and Kim KS (2018): Potential therapeutic antibodies targeting specific adiponectin isoforms in rheumatoid arthritis. Arthritis Res. Ther ; (20): 245.

12. Minamino $\mathrm{H}$, Katsushima $\mathrm{M}$, Yoshida $\mathrm{T}$, Hashimoto M, Fujita Y, Shirakashi M, Yamamoto W, Murakami K, Murata K and Nishitani K (2020): Increased circulating adiponectin is an independent disease activity marker in patients with rheumatoid arthritis: A cross-sectional study using the KURAMA database. Plos one; 3(15): e0229998.

13. Li P, Yang L, Ma CL, Liu B, Zhang X, Ding R and Bi LQ (2015): Low-molecularweight adiponectin is more closely associated with disease activity of rheumatoid arthritis than other adiponectin multimeric forms. Clinical Rheumatology; 6(34): 1025-1030.

14. Veluri G, Vinapamula KS, Rao PVS, Kommireddy S, Manohar SM and Pasupuleti P (2019): Serum adiponectin levels in patients with rheumatoid arthritis.Indian Journal of Rheumatology; 2(14): 119.

15. Zhao Y, Zhang $\mathrm{T}$ and $\mathrm{Wu} \mathrm{J}$ (2020): Association of adiponectin and adiponectin receptor gene polymorphisms with rheumatoid arthritis in a Chinese population. Postgraduate medical journal; 96(1133):149-155.

16. Berg G, Barchuk M, and Miksztowicz V (2019): Behavior of metalloproteinases in adipose tissue, liver and arterial wall: an update of extracellular matrix remodeling, Cells; 8(2): 158.

17. Fassio A, Idolazzi L, Jaber M, Dartizio C, Viapiana O, Rossini M and Gatti D (2016): The negative bone effects of the disease and of chronic corticosteroid treatment in premenopausal women affected by rheumatoid arthritis. Reumatismo. ; 65-71.

18. Mpalaris V, Anagnostis $\mathrm{P}$, Anastasilakis AD, Goulis DG, Doumas A and Iakovou I (2016): Serum leptin, adiponectin and ghrelin concentrations in post-menopausal women: Is there an association with bone mineral density? Maturitas; (88): 32-6.

19. Targońska-Stepniak B, Dryglewska M and Majdan M (2010): Adiponectin and leptin serum concentrations in patients with rheumatoid arthritis. Rheumatology international; 30(6):731-737. 


\section{مستوى مادة الاديبونكتين في الام وعلاقته بهثاثة العظام في المرضى الذين يعانون من مرض الرثيان المفصلي}

المقدمه: الاديبونكتين يصنع ويفرز من الأنسجة الدهنية و أظهرت الدر اسات العلمية الأساسية أن مادة الأديبونيكتين

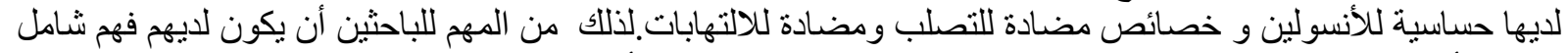

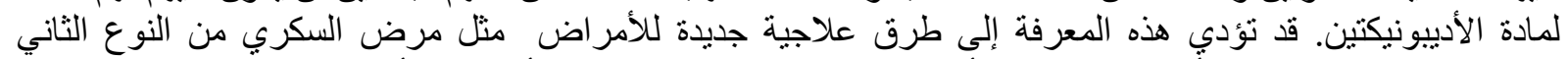

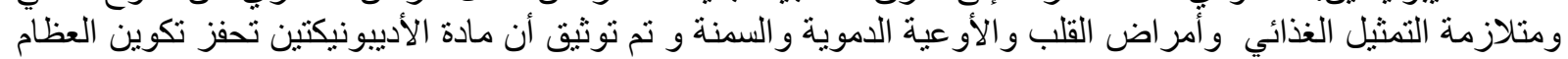

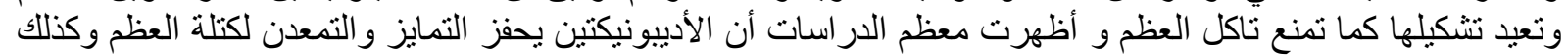

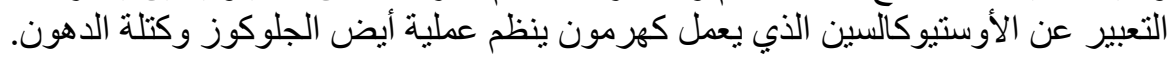

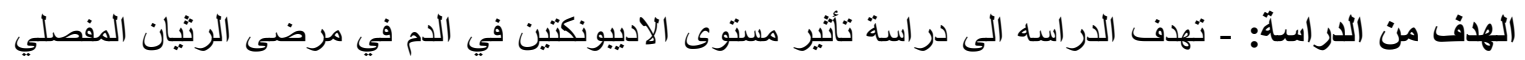

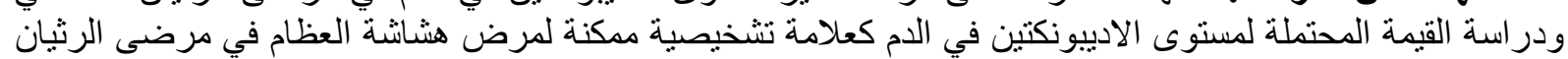
المفصلي .

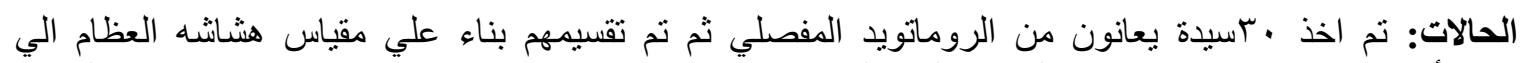

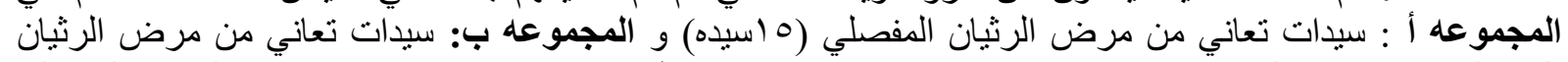

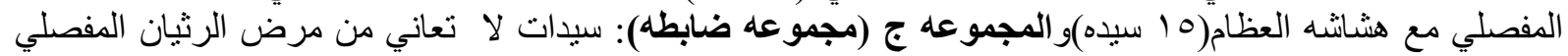

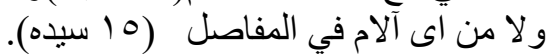

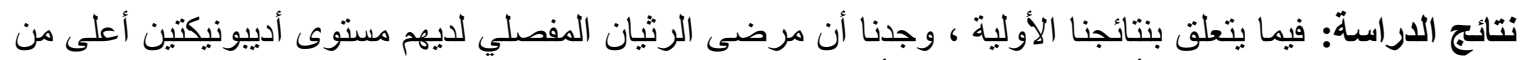

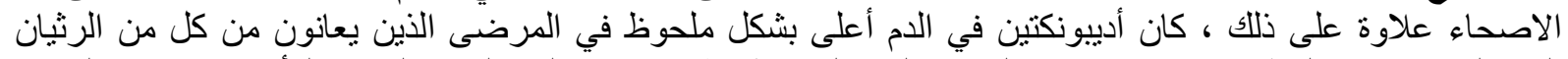

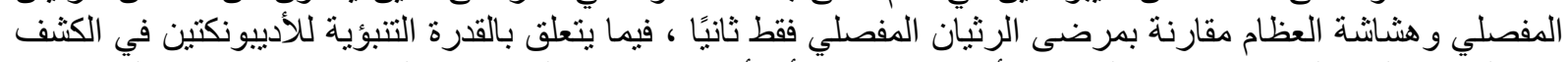

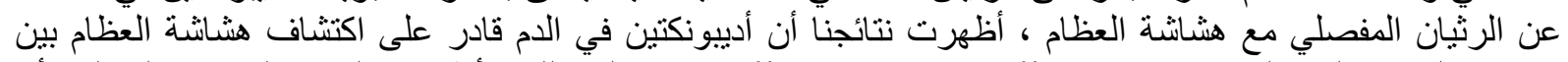

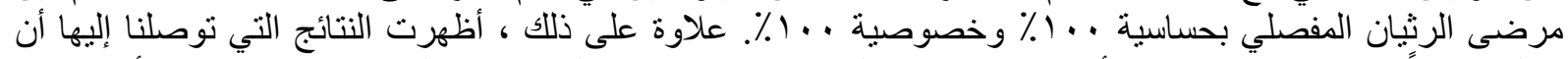

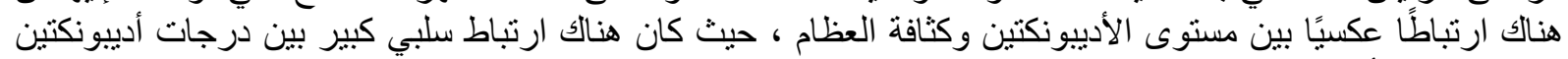

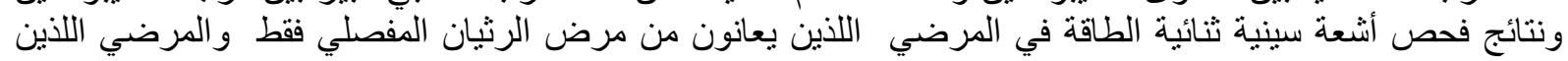

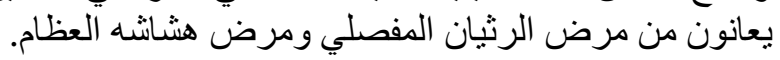

